\title{
The expression of exhaustivity and scalarity in Burmese*
}

\author{
Keely New \\ National University of Singapore \\ Michael Yoshitaka Erlewine \\ National University of Singapore
}

\begin{abstract}
The Burmese particle hma expresses exhaustivity in some contexts but a scalar, even-like meaning in other contexts. We detail the distribution of these uses of $h m a$ and develop a unified semantics. We propose that $h m a$ is a not-at-issue scalar exhaustive, with semantics similar to that proposed for English it-clefts in Velleman, Beaver, Destruel, Bumford, Onea \& Coppock 2012. When hma takes wide scope, it leads to an exhaustive, cleft interpretation which is not scale-sensitive. When hma takes scope under negation, the resulting meaning will have a scale-sensitive felicity condition. We also discuss the semantics of the sentence-final mood marker dar, which we propose is a marker of propositional clefts (Sheil 2016), and its apparent role in the determination of the scope of $h m a$.
\end{abstract}

Keywords: Burmese, focus particle, exhaustivity, scalarity, cleft, propositional cleft

This paper investigates the focus particle hma in Colloquial Burmese which descriptively contributes exhaustivity in some contexts and a scalar meaning in others. In the affirmative declarative sentence in (1), hma has an exhaustive interpretation. In the negative declarative in (2), hma seems to have a scalar interpretation. ${ }^{1}$

(1) Exhaustive hma (cleft):

Aung-ga ye-ko-hma thauq-keh-deh.

Aung-NOM water-ACC-HMA drink-PAST-NONFUT

'It's WATER that Aung drank.'

\section{Scalar hma ('even'-like):}

Aung-ga ye-ko-hma ma-thauq-keh-dar.

Aung-NOM water-ACC-HMA NEG-drink-PAST-DAR

$\approx$ 'Aung didn't even drink WATER.'

*We thank our Burmese speakers and teachers, Phyo Thi Han, Chit Thiri Maung, and Saw Ohnmar Oo, as well as Chris Davis, Hadas Kotek, and Elin McCready, and the audience at SALT 28 for helpful comments and discussion.

${ }^{1}$ The following abbreviations are used in glosses: NOM = nominative, ACC = accusative; NONFUT $=$ non-future, FUT $=$ future; NEG = negation. The mood marker DAR will be discussed in section 4. Here we follow the romanization for Burmese employed in Okell 1994, also discussed in Jenny \& Hnin Tun 2016: 45-47.

(C)2018 New \& Erlewine 
In his reference grammar of Colloquial Burmese, Okell (1969: 284-286) includes two separate lexical entries for " $h m a_{\mathrm{A}}$ " and " $h m a_{\mathrm{B}}$," translated as 'only' and 'even' and corresponding to the uses in (1) and (2), respectively, with no description of the distributions of these two uses.

In this paper we propose a uniform semantics for hma. After some background on the Burmese verbal complex in section 1, we begin in section 2 by describing the environments associated with the apparent exhaustive and scalar uses. We propose in section 3 that hma is a not-at-issue scalar exhaustive with semantics similar to that proposed for English it-clefts in Velleman, Beaver, Destruel, Bumford, Onea \& Coppock 2012. We then discuss the semantics of the sentence-final mood particle dar, which commonly co-occurs with scalar uses of hma, in section 4.

\section{Background: The Burmese verbal complex}

In this section, we give a brief introduction to features of the Burmese verbal complex that will be relevant for our discussion. Burmese is a Tibeto-Burman language spoken in Myanmar, with default SOV word order. The basic verbal complex template in Burmese is given in (3). In particular, we will see in subsequent sections that the choice of sentence-final mood marker will be relevant for describing the distribution of different uses of hma.

\section{(3) Verbal complex template:}

(negation) - verb stem - (past/progressive) - mood

In an affirmative declarative sentence, the verb stem combines with a post-verbal tense/aspect marker, if any, and a sentence-final mood marker. The regular mood markers are the non-future $d e h$ and future meh, illustrated in (4) below. ${ }^{2}$ The use of a mood marker is obligatory.

\section{The basic mood morphemes deh and meh:}

a. Aung-ga ye-ko thauq-keh-*(deh).

Aung-NOM water-ACC drink-PAST-NONFUT

b. thauq-*(meh)

drink-FUT

'Aung drank water.'

'will drink'

Negation in a declarative sentence is expressed by the pre-verbal morpheme $m a$ coupled with the sentence-final negative mood $b u$. The negative mood marker $b u$ takes the place of the final mood markers deh/meh, obscuring the non-future/future distinction. (5) is, however, unambiguously past tense due to the separate keh PAST morpheme.

\footnotetext{
${ }^{2}$ We follow Jenny \& Hnin Tun 2016 in glossing these as non-future vs future, but we note that many other scholars of Burmese describe these markers as realis vs irrealis.
} 
The expression of exhaustivity and scalarity in Burmese

\section{Sentential negation $m a$ triggers $b u$ mood marker:}

a. ma-thauq-keh-\{bu/*deh $\}$

NEG-drink-PAST-NEG

'didn't drink' b. ma-thauq- $\{$ bu/*meh $\}$

NEG-drink-NEG

'will not/does not drink'

One more mood marker, dar, will become important in our discussion below. As we discuss in section 4, the use of dar reflects that the current utterance has a particular status within the organization of the discourse. Like the negative mood marker $b u$, dar takes the mood slot in the verbal template, replacing other markers.

\section{The scalar and exhaustive uses of $\mathrm{hma}$}

The particle $h m a$ appears to give rise to different interpretations in different contexts, as reflected by its variable translations as 'only' or 'even' in Okell 1969: 284-286. In this section, we describe the different environments which elicit these different interpretations. The scalar, 'even'-like interpretation comes about in the presence of local (clause-mate) negation and the mood marker dar; in the absence of these ingredients, hma leads to an exhaustive interpretation.

Before we begin, some discussion is in order regarding these notions of exhaustivity and scalarity. Hma is a constituent focus particle which adjoins to a focus-containing constituent. Let $\mathrm{X}$ be the stated, prejacent value of the focused constituent, and Alt be a contextually-determined set of alternatives to X. An exhaustive use is one which elicits an inference that the alternative propositions with $X$ replaced by any alternative in Alt will be false. A scalar use is one where the felicity of the utterance depends on a contextually-determined ranking of $\mathrm{X}$ with respect to its alternatives in Alt. Examples with exhaustive hma are not sensitive to such contextual ranking of relative likelihood.

Our speakers who are bilingual in Burmese and English translate the exhaustive uses of hma with an English it-cleft or only and translate the scalar uses with English even. (We will argue that the exhaustive use has cleft semantics rather than only semantics, so we will use English it-cleft translations for the exhaustive uses.) However, our description of particular uses as "exhaustive" or "scalar" is not determined by these speakers' English translations, and instead depends on our diagnostics for exhaustivity and scale-sensitivity.

It's important to note that our descriptions of hma as "exhaustive" or "scalar" are descriptive labels which reflect the overall contribution of $h m a$ in a particular example. We will ultimately argue in section 3 that the semantic contribution of hma is in fact uniform across all examples. Part of the challenge, then, which we will meet, is to explain how a uniform semantics leads to these different descriptive behaviors and translations in different contexts. 
We begin with an illustration of the scalar hma. Consider (6). Here we refer to the proposition 'that Aung drank water' without negation as the prejacent. The use of $h m a$ in (6) requires that the prejacent be contextually more likely compared to its alternative (6a) - that it is more likely for Aung to drink water than for Aung to drink something else - and is infelicitous when the prejacent is less likely (6b).

\section{Scalar hma:}

Context: There were many drinks at the party. Aung is a child, so out of all the drinks, it is expected that Aung will drink water. It would be less likely or more noteworthy for Aung to drink beer.
a. Aung-ga ye-ko-hma ma-thauq-keh-dar.
Aung-NOM water-ACC-HMA NEG-drink-PAST-DAR
$\approx$ 'Aung didn't even drink WATER.'
b. \# Aung-ga biya-ko-hma ma-thauq-keh-dar. Aung-NOM beer-ACC-HMA NEG-drink-PAST-DAR Intended: $\approx$ \# 'Aung didn't even drink BEER.'

This scalar behavior of $h m a$ appears to be similar to that of so-called "scale-reversed" even (Karttunen \& Peters 1979), which we use in the English translations in (6).

An example of the exhaustive use of hma is presented in (7). (7B) expresses that Aung drank water and that he drank nothing else, disallowing the continuation that Aung also drank beer. In example (7B), the prejacent 'that Aung drank water' is a relatively likely possibility given the context. But unlike in (6), the use of $h m a$ in (7) is not sensitive to the relative likelihood of the prejacent, as indicated by the felicity of (7B') where the prejacent is relatively unlikely.

\section{Exhaustive hma (cleft):}

Context: Aung is a child. It is more likely that he will drink water than beer.

A: I wonder what Aung drank. Maybe it was juice.

B: (Ma-houq-bu,) Aung-ga ye-ko-hma thauq-keh-deh. NEG-right-NEG Aung-NOM water-ACC-HMA drink-PAST-NONFUT

'(No,) it's WATER that Aung drank.' \# ...Aung also drank beer.

B': (Ma-houq-bu,) Aung-ga biya-ko-hma thauq-keh-deh. NEG-right-NEG Aung-NOM beer-ACC-HMA drink-PAST-NONFUT

'(No,) it's BEER that Aung drank.' \# ...Aung also drank water.

What determines whether a particular use of hma will be interpreted as scalar or exhaustive? The examples which yield these different meanings in (6) and (7) above differ in two ways: the scalar (6) is negated and ends with the dar mood 
The expression of exhaustivity and scalarity in Burmese

marker, whereas the exhaustive (7) is affirmative with default (here, non-future) mood markers. Descriptively, the local sentential negation and the sentence-final mood marker dar together yield the scalar interpretation.

We might hypothesize that any use of hma in a negative clause is necessarily scalar. However, the exhaustive use is also compatible with negation. Consider example (8). Hma here is interpreted as exhaustive, scoping over negation.

\section{(8) Exhaustive hma with local negation:}

Aung-ga ye-ko-hma ma-thauq-keh-bu.

Aung-NOM water-ACC-HMA NEG-drink-PAST-NEG

'It's WATER that Aung didn't drink.'

exhaustive $>$ NEG

Recall that sentential negation $m a$ triggers the use of the negative mood marker $b u$ which is used here in (8). (8) contrasts minimally with the scalar hma examples in (6) in this choice of mood marker. With negation and the default negative mood marker $b u$, we yield exhaustive hma scoping over negation in (8). With negation and the dar mood marker, we yield the scale-sensitive interpretation observed in (6).

Next we turn to the behavior of hma embedded under a higher, non-clause-mate negation, in example (9). The interpretation of (9) is unambiguously an embedded exhaustive, rather than a scalar use. This teaches us that local sentential negation (in addition to dar) is necessary for the scalar use of hma in (6).

Hma under higher negation is exhaustive:

[CP Aung-ga ye-ko-hma thauq-keh-deh/dar] ma-houq-bu.

Aung-NOM water-ACC-HMA drink-PAST-NONFUT/DAR NEG-right-NEG

literally 'It's false [CP that Aung drank water-hma].'

a. 'It isn't WATER that Aung drank.'

$\rightsquigarrow$ Aung drank something.

$\rightsquigarrow$ if Aung drank water, he didn't drink anything else.

$y \rightarrow$ Aung drank water.

b. * 'Aung didn't even drink WATER.'

Example (9) also shows that the exhaustive inference of hma is not at-issue content. From this sentence, we infer commitments that Aung drank something and that water would be an exhaustive answer to 'What did Aung drink?', but not that Aung drank water. The prejacent 'that Aung drank water' is (9)'s at-issue content, with the existential and (conditional) exhaustivity inferences projecting out of the higher negation. This accords with the behavior of English it-clefts; see especially discussion in Büring 2011, Büring \& Križ 2013, and Velleman et al. 2012. The behavior in (9) is clearly unpredicted if $h m a$ were an at-issue exhaustive with a prejacent presupposition, as in English only (Horn 1969). 
New \& Erlewine

\section{Proposal}

We propose a uniform semantics for $h m a$ as a not-at-issue scalar exhaustive, similar to the semantics proposed for English it-clefts in Velleman et al. 2012. We analyze the descriptively scalar and exhaustive uses of hma observed above as the result of a scope ambiguity: When the scalar exhaustive $h m a$ scopes under negation, the resulting meaning will be a scalar meaning, sensitive to the relative likelihood of the prejacent with respect to its alternatives. The interaction with the sentence-final mood marker dar will be discussed further in section 4 .

$H m a$ encliticizes to the focus-containing constituent but takes propositional scope at LF within the same clause. ${ }^{3}$ Let $p$ be the intension of hma's sister at $\mathrm{LF}$, the prejacent proposition. $C$ is the set of focus alternatives to $p$ (Rooth 1992)

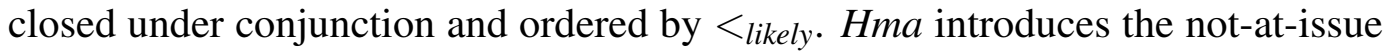
requirement in (10), which for concreteness we describe as a presupposition.

\section{Presupposition of hma:}

$$
h m a_{C}(p)\left(w^{\star}\right) \rightsquigarrow \forall q \in C\left[\left(q<_{\text {likely }} p\right) \rightarrow \neg q\left(w^{\star}\right)\right]
$$

"No less likely alternative is true."

This meaning in (10) is a version of the MAX exhaustivity operator proposed by Velleman et al. (2012) for the not-at-issue part of English it-clefts, based in turn on Beaver \& Clark 2008 and Coppock \& Beaver 2014. As we will see, an important feature of our proposal is the requirement that the alternatives considered, $C$, be closed under conjunction.

We first consider the effect of $h m a$ in a simple affirmative sentence. We will first model the behavior of example (1/7B) above. Suppose the set of alternatives $C$ contains two atomic alternatives, $A=$ 'that Aung drank water' and $B=$ 'that Aung drank beer,' as well as their conjunction $A \wedge B$. The conjunctive alternative is necessarily less likely than each atomic alternative. In addition, given the context in (7) where Aung is a child, $A>_{\text {likely }} B$.

$$
\begin{aligned}
& \text { Alternatives in context (7): } \\
& \begin{array}{c}
C=\{A, B, A \wedge B\} \\
A=\text { 'that Aung drank water' }>_{\text {likely }} B=\text { 'that Aung drank beer' } \\
\checkmark_{\text {likely }}
\end{array}
\end{aligned}
$$

$A \wedge B=$ 'that Aung drank water and beer'

\footnotetext{
${ }^{3}$ This can be thought of as hma moving from its pronounced position, in a clause-bound fashion, or as hma agreeing with a covert HMA on the clausal spine, with this dependency being clause-bound.
} 
The expression of exhaustivity and scalarity in Burmese

At LF, hma takes the proposition 'that Aung drank [water] $]_{\mathrm{F}}$ as its sister, so the prejacent $p=A$. Given the alternatives $C$ from (11), $h m a$ will assert the prejacent $A$ and presuppose that no less likely alternative is true. There are two less likely alternatives, $B$ and $A \wedge B$, resulting in the presupposition in (12b):

\section{(12) Computing hma in (1/7B):}

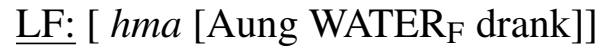

a. asserts: $A$

b. $\quad$ presupposes: $h m a_{C}(A)\left(w^{\star}\right)=\neg(A \wedge B) \wedge \neg B \quad \Rightarrow$ exhaustive (cleft)

We predict that (1/7B) will assert the prejacent $A$ and presuppose the negations of $B$ and $A \wedge B$. The result is an exhaustive claim (cleft): Aung drank water, and could not have drank anything else.

Now consider instead if the prejacent were $B$, 'that Aung drank beer,' in the same context with alternatives $C$. This corresponds to the use of $h m a$ in (7B'), with the prejacent referring to beer. In this case, when computing the presupposition of hma, there is only one alternative that is less likely than the prejacent: the conjunctive alternative $A \wedge B$.

(13) Computing hma in (7B'):

LF: [ hma [Aung BEER $\mathrm{F}$ drank]]

a. asserts: $B$

b. $\quad$ presupposes: $h m a_{C}(B)\left(w^{\star}\right)=\neg(A \wedge B) \quad \Rightarrow$ exhaustive (cleft)

This results in the assertion of $B$ and the presupposition of $\neg(A \wedge B)$. However, taken together, these requirements entail that $A$ must be false. In this way, the same scalar meaning for $h m a$ in (10) results in an exhaustive interpretation for both a more likely prejacent $(A)$ and a less likely prejacent $(B)$.

Next we consider the behavior of hma in the presence of local negation. We propose that both scope possibilities $h m a>$ NEG and NEG $>h m a$ are possible at LF. Hma > NEG will yield a cleft with negation in its scope, as in example (8) above. In contrast, NEG > hma will yield the scalar use of hma described above. In section 2 above, we showed that the scalar and exhaustive interpretations of hma in the presence of local negation correlate with the presence or absence of the sentence-final dar mood marker, respectively. In section 4, we will argue that this apparent correlation is governed pragmatically, not syntactically. In the remainder of this section, then, we will simply consider the contribution of hma with both scope possibilities, $h m a>$ NEG and NEG $>h m a$, and return to the connection to dar in section 4.

We begin first with the consideration of $h m a>$ NEG, which will derive a cleft with negation in its scope, as in (8). Following the notation from above ( $A=$ 'that 
Aung drank water,' $B=$ 'that Aung drank beer'), $C$ will now include the negated atomic alternatives $\neg A$ and $\neg B$, as well as the conjunctive alternative $\neg A \wedge \neg B$. Following the context in (7), the atomic alternatives are ordered $\neg A<_{\text {likely }} \neg B$ and the conjunctive alternative is again less likely than each atomic alternative.

$$
\begin{aligned}
& \text { Alternatives in context (7), with negation in scope: } \\
& \begin{array}{c}
C=\{\neg A, \neg B, \neg A \wedge \neg B\} \\
\neg A=\text { 'Aung didn't drink water' } \quad<_{\text {likely }} \quad \neg B=\text { 'Aung didn't drink beer' } \\
\qquad \downarrow_{\text {likely }},{ }_{\text {likely }} \\
\neg A \wedge \neg B=\text { 'Aung didn't drank water and didn't drink beer' }
\end{array}
\end{aligned}
$$

The use of $h m a$ with prejacent $\neg A$ or $\neg B$ will both yield an exhaustive interpretation, just as hma did without negation in (12-13) above. Here we illustrate the case of $p=\neg A$, corresponding to example (8) above. The presupposition of $h m a$ will require that the conjunctive alternative $\neg A \wedge \neg B$ be false. Together with the assertion that Aung didn't drink water $(\neg A)$, we yield the exhaustive claim that water is the only thing that Aung didn't drink.

\section{(15) Computing hma in (8), hma > NEG:}

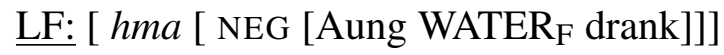
a. asserts: $\neg A$
b. $\quad$ presupposes: $h m a_{C}(\neg A)\left(w^{\star}\right)=\neg(\neg A \wedge \neg B)$
$\Rightarrow$ cleft $>$ NEG

We similarly yield an exhaustive claim if $h m a$ applies to $\neg B$ :

Computing $h m a, h m a>$ NEG, with prejacent $\neg B$ :

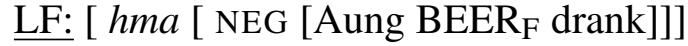

a. asserts: $\neg B$

b. $\quad$ presup.: $h m a_{C}(\neg B)\left(w^{\star}\right)=\neg(\neg A) \wedge \neg(\neg A \wedge \neg B) \quad \Rightarrow$ cleft $>$ NEG

Following the logic of (12-13) above, hma in both (15) and (16) result in an exhaustive claim of their prejacent. Hma with scope over negation is compatible with both more likely alternatives and less likely alternatives, i.e. it is not scale-sensitive.

Next we consider the contribution of hma with scope under negation. In contrast to the uses of hma modeled above, which uniformly contribute exhaustivity, we argue that the NEG > hma configuration results in the descriptively scale-sensitive use of hma akin to scale-reversed 'even.' Concretely, we will model the examples in (6) above. Because negation is outside of the scope of $h m a$, the relevant set of 
The expression of exhaustivity and scalarity in Burmese

alternatives $C$ will be without negation as in (11) above, repeated here in (17). The two atomic alternatives are again ordered with $A>$ likely $B$, since the context in (6) assumes Aung to be a child and therefore more likely to drink water than beer, just as in (7) above.

Alternatives in context (6), below negation:

$$
\begin{gathered}
C=\{A, B, A \wedge B\} \\
A=\text { 'that Aung drank water' }>_{\text {likely }} \quad B={ }^{\text {'that Aung drank beer' }} \\
\checkmark_{\text {likely }}
\end{gathered}
$$

$A \wedge B=$ 'that Aung drank water and beer'

We now compute the LFs for (6), with higher negation, with $A$ and $B$ in turn as the prejacent. Since the alternatives $C$ are the same as in the computation of (12-13) above, the presuppositions introduced by $h m a$ will be equivalent. With $A$ as the prejacent, hma presupposes the negation of $B$ as well as the negation of the conjunction $A \wedge B(18 \mathrm{~b})$. With $B$ as the prejacent, hma simply presupposes the negation of the conjunctive alternative $A \wedge B$, as this is the only alternative that is less likely than the prejacent in $C$ (19b). However, in contrast to (12-13) above, the assertions (respectively, $A$ and $B$ ) will be negated above $h m a$. Since presuppositions project through negation, the presuppositions in (18-19) - equivalent to those in (12-13) - will project unaffected.

\section{Computing $h m a$ in (6a), NEG > hma:}

$\underline{\mathrm{LF}}$ : [ NEG [ hma [Aung WATER $\mathrm{F}$ drank]]]

a. asserts: $\neg A$

b. $\quad$ presupposes: $h m a_{C}(A)\left(w^{\star}\right)=\neg(A \wedge B) \wedge \neg B$

(19) Computing hma in (6b), NEG > hma:

LF: [ NEG [ hma [Aung BEER $F$ drank]]]

a. asserts: $\neg B$

b. $\quad$ presupposes: $h m a_{C}(B)\left(w^{\star}\right)=\neg(A \wedge B)$

We consider each of these results in turn. (18) illustrates the case where the prejacent is $A=$ 'that Aung drank water,' the contextually more likely alternative. The presupposition of $h m a$ here requires that the contextually less likely alternative $B$ be false. In contrast, consider the contribution of hma in (19). Here the predicted presupposition $\neg(A \wedge B)$ is entailed by and logically weaker than the assertion $\neg B$.

We adopt the view that the use of focus particles such as hma is governed by a Non-Vacuity condition such as (20) from Crnič 2011. 


\section{(20) The Principle of Non-Vacuity (Crnič 2011: 110):}

The meaning of a lexical item used in the discourse must affect the meaning of its host sentence (either its truth-conditions or its presuppositions).

To evaluate a use of $h m a$ for Non-Vacuity, we compare its overall meaning contribution to that of the utterance without hma. The addition of hma in (18) is contentful, as it expresses presuppositional content beyond the commitments made by the speaker in the assertion. The same goes for the use of hma in the descriptively exhaustive uses in (12-13) above. However, the addition of hma is not contentful in (19): The presupposition introduced by $h m a, \neg(A \wedge B)$, is logically weaker than the assertion and therefore its addition in (19) is uninformative. The use of $h m a$ in (6b/19) is ruled ungrammatical by a Non-Vacuity principle as in (20).

The end result is that the felicitous use of hma scoping under negation will depend on the relative position of the prejacent on the contextually-determined scale of likelihood. Hma is infelicitous when the prejacent is the least likely alternative, as in (19). The direction of this asymmetry also explains why, in the basic case, scalar hma is naturally translated into English with scale-reversed even.

It's worth noting that it's not always the case that hma within the scope of negation leads to such a scale-sensitive asymmetry on its felicitous use. See for example (9) above, where hma is in an embedded clause, under matrix negation. The variant of (9) with 'beer' in place of the focus 'water' is also grammatical, in the same context. We propose that Non-Vacuity is evaluated cyclically, at the completion of each clause or phase. In example (9), the addition of hma is meaningful at the embedded CP level, regardless of the choice of focus, licensing the use of hma. This whole meaning is then negated by the higher negation.

Together with an independently motivated Non-Vacuity condition on the use of focus particles (20), our uniform analysis for the semantics of hma as a not-at-issue scalar exhaustive successfully derives the distribution of felicitous and infelicitous uses of $h m a$, including its uses which may at first glance be described as "exhaustive" or "scalar." These patterns are summarized in the table below.

$$
\begin{aligned}
& \text { Summary: } \\
& C=\{A, B, A \wedge B\}, A>_{\text {likely }} B
\end{aligned}
$$

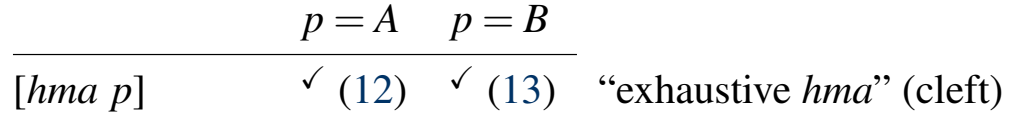

$$
\begin{aligned}
& \text { [hma [NEG } p \text { ]] } \checkmark \text { (15) } \checkmark \text { (16) “exhaustive hma" (cleft) > NEG } \\
& \text { [NEG [hma p]] } \checkmark(18) *(19) \text { "scalar hma" }
\end{aligned}
$$


The expression of exhaustivity and scalarity in Burmese

\section{The role of sentence-final dar}

In this section we discuss the interaction of sentence-final mood markers with the interpretation of hma. As we saw in section 2, hma in the presence of local negation may be an exhaustive particle (cleft) with scope over negation or a scalar particle akin to English scale-reversed even. In the basic examples considered in section 2 — repeated in (23) and (24) — this choice of interpretation appears to correlate with the presence or absence of the mood marker dar. Under our unified analysis of the focus particle $h m a$ as a scalar exhaustive, presented in the previous section, these two different uses reflect a scope ambiguity at LF. This is summarized in (22).

(22) Two interpretations for hma with local negation:

$\begin{array}{lllll} & \text { description } & \text { mood marker } & \text { LF } & \\ \text { a. "scalar } h m a " & \text { dar } & \text { NEG }>h m a & \text { (23) } \\ \text { b. "exhaustive } h m a ">\text { NEG } & \text { default }(b u) & h m a>\text { NEG } & \text { (24) }\end{array}$

Scalar hma:

Aung-ga ye-ko-hma ma-thauq-keh-dar.

Aung-NOM water-ACC-HMA NEG-drink-PAST-DAR

'Aung didn't even drink WATER.'

Exhaustive hma > NEG:

Aung-ga ye-ko-hma ma-thauq-keh-bu.

Aung-NOM water-ACC-HMA NEG-drink-PAST-NEG

'It's WATER that Aung didn't drink.'

The question we address in this section is how to explain this correlation between the presence or absence of sentence-final dar and the LF scope of hma. We consider two hypotheses. The first is that this correlation is enforced syntactically: The presence of dar in (22a) (somehow) syntactically blocks hma from scoping over negation, but hma otherwise obligatorily scopes over negation, as in (22b). The second is that this correlation is more indirect, based on the semantics of dar: dar appears in utterances with a particular semantics/pragmatics, and scalar hma (NEG > hma) supports its use, whereas exhaustive hma (in general) does not.

We will argue for this second, indirect approach to the observed correlation. We propose that dar is a marker for propositional clefts (PC). In previous work, Kato (1998: 88-89) notes that utterances with dar are similar to Japanese -no-da PCs, and Andrew Simpson (p.c.) notes that dar is similar to Mandarin Chinese shi...de PCs. In her study of PCs - both in depth in Scottish Gaelic and more broadly, cross-linguistically - Sheil (2016) proposes that PCs are utterances the revise the "line of inquiry." In the Roberts 1996/2012 Question Under Discussion (QUD) 
tradition, PCs address a super-question of the immediate QUD or a new sub-question thereof (i.e. a sister question of the original immediate QUD). See Sheil 2016 chapter 3 for detailed discussion of and motivation for these constraints on PC congruence.

We substantiate this proposal for dar through data on the (in)felicity of dar for different types of discourse moves, for utterances without hma. We first note that the use of dar is inappropriate for direct answers to existing questions. This is particularly clear with the explicit question in (25A), which can be answered in (25B) using the default mood marker (here, non-future deh) but not with dar.

\section{Dar is inappropriate for direct answers to questions:}

A: What did Su drink?

B: Su-ga ye-ko thauq-keh- ${ }^{\checkmark}$ deh/*dar. Su-NOM water-ACC drink-PAST ${ }^{\checkmark}{ }^{\vee}$ NONFUT $/{ }^{*}$ DAR

'Su drank water.'

In (25), 'What did Su drink?' was clearly the immediate QUD. A direct answer to this question does not license the use of the PC marker, dar.

In contrast, the use of dar is natural in the correction in (26). In such an utterance where it is licensed, its use is judged as near-obligatory.

\section{Dar is appropriate for corrections:}

A: Su drank beer.

B: Ma-houq-bu, Su-ga ye-ko thauq-keh-*deh ${ }^{\sqrt{ }}$ dar. NEG-right-NEG Su-NOM water-ACC drink-PAST-* NONFUT ${ }^{\checkmark}{ }^{2}$ DAR

'No, Su drank water.'

Here, B disputes A's claim that Su drank beer and instead offers the assertion that $\mathrm{Su}$ drank water instead. B shifted to a new QUD, that of whether Su drank water or not.

The organization of this discourse is illustrated in discourse-tree (or $d$-tree; see Büring 2003, also Constant 2014, Rojas-Esponda 2014, Sheil 2016) form in (27). Questions in the d-tree end with? and implicit moves are in parentheses.

\section{D-tree for (26):}

(What did Su drink?)

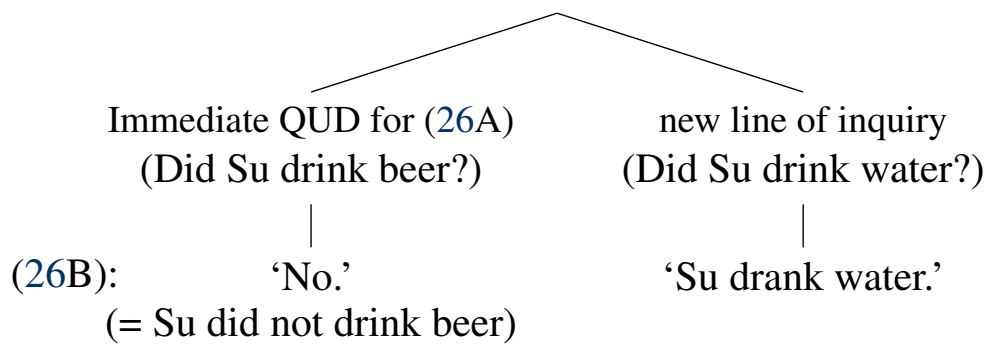


The expression of exhaustivity and scalarity in Burmese

Speaker A addresses the implicit polar question 'Did Su drink beer?' Speaker B addresses this immediate QUD with a contrasting answer, 'No,' and then asserts that 'Su drank water' instead. This assertion is not an answer to the original immediate QUD, but instead addresses an (implicit) sister question, 'Did Su drink water?' (These two questions are sister questions in that they are both natural sub-questions of the implicit super-question 'What did Su drink?') B's shift to address a new sister question (the new "line of inquiry") makes the assertion that Su drank water here a PC and thus marked by dar. In contrast, the same assertion that Su drank water in the discourse (25) above, where it addresses the immediate QUD, does not count as a PC and dar cannot be used.

With this basic formal proposal for the use of dar in place, we return to the role of dar in the determination of scalar vs exhaustive uses of hma in the presence of local negation. Consider first the scalar use, which we describe as the effect of hma scoping under negation: NEG > hma. Our canonical example is repeated here:

(28) Scalar hma:

Aung-ga ye-ko-hma ma-thauq-keh-dar.

Aung-NOM water-ACC-HMA NEG-drink-PAST-DAR

'Aung didn't even drink WATER.'

Now we consider the types of discourses which support the felicitous use of scalar $h m a$, i.e. NEG $>$ hma. Although the assertive content of (28) is simply that Aung didn't drink water, (28) is judged as an unnatural reply to the polar question 'Did Aung drink water?' (29a). Instead, (28) is most natural when the immediate QUD is a sister-question such as 'Did Aung drink beer?' (29b) or a super-question such as 'What did Aung drink?' (29c). ${ }^{4}$

\section{(29) Preceding discourses for scalar hma (28):}

a. A: Did Aung drink water?

B: \# (No,) (28)

b. A: Did Aung drink beer? / (I think) Aung drank beer.

B: ${ }^{\checkmark}$ No, (28)

c. A: What did Aung drink? / I wonder what Aung drank.

B: $\checkmark(28)$

In all of these contexts, then, the utterance of scalar hma does not address or resolve what was the immediate QUD, but instead contributes to the conversation by

\footnotetext{
${ }^{4}$ Interestingly, in our judgment, the English translation for (28) using scale-reversed even, 'Aung didn't even drink WATER,' also follows the pattern of felicity in (29).
} 
addressing a sister-question (in (29b)) or a sub-question (in (29c)) of the immediate QUD. In other words, scalar hma with NEG > hma naturally addresses a new line of inquiry in Sheil's terms, and is thus a PC in both (29b) and (29c). We predict the use of dar in each of these cases.

We can potentially go one step further and predict the use of dar as a necessary consequence of an utterance with NEG $>$ hma. Suppose we adopt the proposal from Beaver \& Clark 2008 that sets of focus alternatives ( $C$ in (10)) must be congruent to a QUD. With NEG > hma scope, the negative assertion necessarily does not directly address the active QUD whose alternatives hma quantifies over. The utterance instead addresses a new line of inquiry, making it a PC and thus marked with dar. To our knowledge, all uses of scalar hma (NEG > hma) indeed do cooccur with dar, supporting this stronger approach, but we believe a wider range of data must be considered first before establishing and motivating this stronger generalization. We leave this in-depth investigation for future work.

In contrast, consider the use of $h m a$ with $h m a>$ NEG scope. As we saw above, this is the interpretation of example (8/24), repeated here as (30), with the default negative mood marker $b u$ in place of dar.

Exhaustive hma > NEG:

Aung-ga ye-ko-hma ma-thauq-keh-bu.

Aung-NOM water-ACC-HMA NEG-drink-PAST-NEG

'It's WATER that Aung didn't drink.'

Our own proposal for the semantics of $h m a$ in (10) mirrors the proposal for the presuppositions of English it-clefts in Velleman et al. 2012. As Velleman et al. discuss, a central property of clefts is that they address and fully resolve an existing QUD. A natural context for the use of (30) is a discourse with the immediate QUD 'What didn't Aung drink?' The utterance of (30) offers an exhaustive answer to this QUD, terminating this line of inquiry. As no new lines of inquiry are implicitly raised in this discourse move, (30) is not a PC and thus resists the use of dar.

Support for this view comes from the fact that exhaustive hma with scope over local negation - i.e. the LF for (30) - can in fact be used with dar if the discourse independently supports the utterance's status as a PC. This is illustrated in (31). Here, speaker A claims that Aung didn't drink beer. Speaker B disagrees with this claim with 'No' and then asserts that 'it's water that Aung didn't drink' with hma > NEG: exhaustive hma with negation in its scope. 
The expression of exhaustivity and scalarity in Burmese

\section{(31) Exhaustive hma > NEG with dar:}

A: Aung didn't drink beer.

B: Ma-houq-bu, Aung-ga ye-ko-hma ma-thauq-keh-dar. NEG-right-NEG Aung-NOM water-ACC-HMA NEG-drink-PAST-DAR 'No, it's WATER that Aung didn't drink.' cleft > NEG

Notice that the second sentence in (31) uses the dar mood marker and is thus stringidentical to (28), but has the interpretation of the cleft with negation in its scope in (30). The additional supporting discourse context allows for this interpretation in (31), even though by default the same string in (28) is interpreted with NEG $>$ hma scope, descriptively as an instance of scalar hma. The availability of this interpretation in (31) is an argument against any approach that predicts a strict, one-to-one relationship between the use of dar and hma taking narrow scope under negation, such as the syntactic approach briefly sketched above.

Our proposal explains the use of dar in (31B). The relevant discourse moves in (31) are illustrated in the d-tree in (32) below. We start with A's statement. This addresses the implicit polar question 'Did Aung not drink beer?' Speaker B addresses this immediate QUD with 'No,' and then shifts to a new line of inquiry: 'What did Aung not drink?', which is a super-question of the original immediate QUD. Using the hma cleft, B gives an exhaustive answer to this super-question: 'It's WATER that Aung didn't drink.'

D-tree for (31):

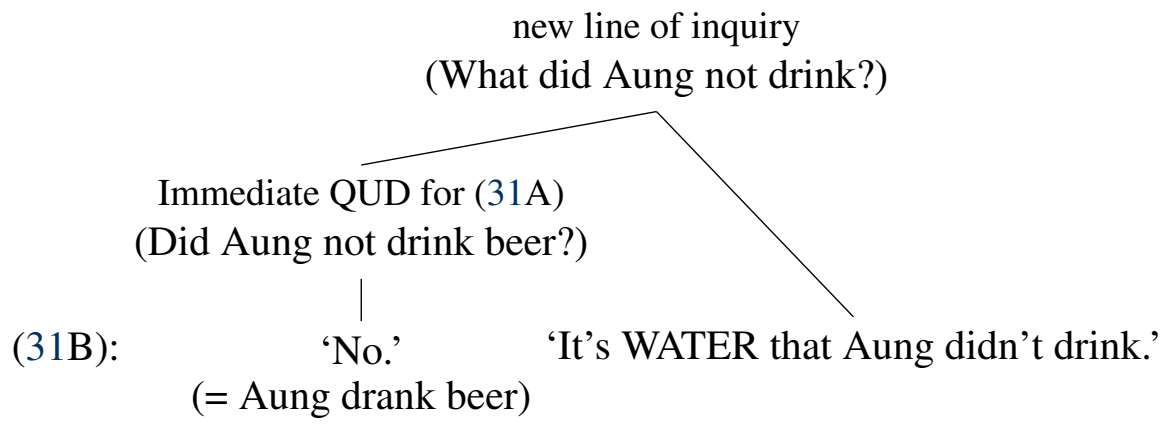

As this cleft 'It's WATER that Aung didn't drink' addresses a new line of inquiry, it counts as a PC and thus licenses dar.

In this section we addressed the role of the sentence-final mood marker dar in the interpretation of $h m a$ in the presence of local negation. In this data on the behavior of $h m a$ as analyzed in section 3, there is a correlation between the presence or absence of dar and the scope of hma above or below negation, respectively. Under the approach laid out here, this correlation is epiphenomenal. The mood marker dar 
is a marker of PCs, which indicate that the utterance addresses a new line of inquiry (Sheil 2016). Utterances with NEG > hma naturally — or perhaps necessarily address a new line of inquiry, explaining the appearance of dar in such examples. With $h m a>$ NEG scope, the utterance is a cleft, fully resolving a QUD (Velleman et al. 2012), which will often be the immediate QUD. However, this correlation is not absolute. As predicted by our account, in a richer discourse where the cleft resolves a new line of inquiry, $h m a>$ NEG interpretation is compatible with the use of dar.

\section{Conclusion}

In this paper we investigated the semantics of the Burmese focus particle hma. Descriptively, hma appears to have two distinct uses, as an exhaustive particle or a scalar particle, with Okell 1969: 284-286 offering both English only and even as translations. We described the distribution of these two apparently distinct uses and offered a uniform analysis for hma as a not-at-issue scalar exhaustive, similar to the analysis of cleft semantics in Velleman et al. 2012. On the surface, hma is a constituent focus particle that encliticizes to a focus-containing phrase but takes propositional scope at LF. The scale-sensitive use of hma comes about when hma scopes under negation, whereas hma taking widest scope leads to exhaustive, cleft semantics.

Burmese hma shows that a common core may underly descriptively "exhaustive" particles, translated as a cleft or only, and descriptively "scalar" particles which might be translated with English even. The analysis here may also extend to similar particles such as Blackfoot-ikak, which Bliss 2010 describes as having a distribution similar or identical to what we show for Burmese hma. The connection between scalar and exhaustive particles has also been discussed more generally in recent work by Greenberg (2018). Such case studies motivate future work which explores a shared, scalar core to such meanings.

Describing the distribution of these two readings of Burmese hma also necessitated a better understanding of the mood marker dar. Following previous descriptions, we analyze dar as a marker for propositional clefts, which reflect a discourse move where a new line of inquiry is addressed (Sheil 2016). We showed that this formal pragmatic description of the description of dar can explain the apparent correlation between the use of dar and the narrow scope of hma, as well as its exceptions.

There is one additional use of $h m a$ which we have not discussed here due to limitations of space. Hma can be used in combination with wh-phrases to form negative polarity items (NPIs). For example, in (33), 'which apple(s)' with hma forms the NPI 'any apple.' 
The expression of exhaustivity and scalarity in Burmese

\section{(33) Wh-hma NPI:}

nga-ga beh-panthi-ko-hma ma-yu-keh-bu.

1-NOM WH-apple-ACC-HMA NEG-take-PAST-NEG

'I didn't take any apple(s).'

The connection between NPIs - and in particular, wh-NPIs - and scalar particles has been documented in a range of previous work (Lee \& Horn 1995; Lahiri 1998; Erlewine \& Kotek 2016; a.o.). In future work, we will present a full analysis of these wh-hma NPIs which capitalizes on the scalar semantics for hma established here.

\section{References}

Beaver, David Ian \& Brady Clark. 2008. Sense and Sensitivity: How Focus Determines Meaning. Wiley-Blackwell.

Bliss, Heather. 2010. Blackfoot -ikak: A case study of only and even. Presented at the Meeting of Semanticists Active in Canada (MOSAIC) 2 at McGill University.

Büring, Daniel. 2003. On D-trees, beans, and B-accents. Linguistics and Philosophy 26. 511-545.

Büring, Daniel. 2011. Conditional exhaustivity presuppositions in clefts (and definites). Manuscript, University of Vienna, July 2011. https://semanticsarchive. net/Archive/DQxOTVjY/Buring.clefts.2011.pdf.

Büring, Daniel \& Manuel Križ. 2013. It's that, and that's it! Exhaustivity and homogeneity presuppositions in clefts (and definites). Semantics \& Pragmatics 6(6). 1-29.

Constant, Noah. 2014. Contrastive topic: meanings and realizations. Amherst, MA: University of Massachusetts Amherst PhD dissertation.

Coppock, Elizabeth \& David Ian Beaver. 2014. Principles of the exclusive muddle. Journal of Semantics 31. 371-432.

Crnič, Luka. 2011. Getting even. Cambridge, MA: Massachusetts Institute of Technology $\mathrm{PhD}$ dissertation.

Erlewine, Michael Yoshitaka \& Hadas Kotek. 2016. Even-NPIs in Dharamsala Tibetan. Linguistic Analysis 40(3-4). 129-165.

Greenberg, Yael. 2018. Even and only: Arguing for parallels in scalarity and in constructing focus alternatives. Presented at North East Linguistics Society (NELS) 49.

Horn, Laurence Robert. 1969. A presuppositional analysis of only and even. In Robert I. Binnick, Alice Davison, Georgia M. Green \& Jerry L. Morgan (eds.), Chicago Linguistic Society (CLS) 5, 98-107.

Jenny, Mathias \& San San Hnin Tun. 2016. Burmese: A Comprehensive Grammar. Routledge. 
Karttunen, Lauri \& Stanley Peters. 1979. Conventional implicature. In Choon-Kyu Oh \& David A. Dinneen (eds.), Syntax and Semantics, Volume 11: Presupposition, 1-56. Academic Press.

Kato, Atsuhiko. 1998. Ekusupuresu Birumago [Express Burmese]. Tokyo: Hakusuisha.

Lahiri, Utpal. 1998. Focus and negative polarity in Hindi. Natural Language Semantics 6. 57-123.

Lee, Young-Suk \& Laurence Horn. 1995. Any as indefinite plus even. Manuscript, Yale University, May 1995.

Okell, John. 1969. A Reference Grammar of Colloquial Burmese. Oxford University Press.

Okell, John. 1994. Burmese: An Introduction to the Spoken Language. Northern Illinois University, Center for Southeast Asian Studies.

Roberts, Craige. 1996/2012. Information structure in discourse: Towards an integrated formal theory of pragmatics. In Jae-Hak Yoon \& Andreas Kathol (eds.), Papers in Semantics, vol. 49 OSU Working Papers in Linguistics, Reprinted in Semantics \& Pragmatics 5(6), 1-69, 2012.

Rojas-Esponda, Tania. 2014. A discourse model for überhaupt. Semantics \& Pragmatics 7(1). 1-45.

Rooth, Mats. 1992. A theory of focus interpretation. Natural Language Semantics 1. 75-116.

Sheil, Christine M. 2016. Scottish Gaelic clefts: Syntax, semantics, and pragmatics. Berkeley, CA: University of California, Berkeley PhD dissertation.

Velleman, Leah, David Ian Beaver, Emilie Destruel, Dylan Bumford, Edgar Onea \& Liz Coppock. 2012. It-clefts are IT (inquiry terminating) constructions. In Semantics and Linguistic Theory (SALT) 22, 441-460.

Keely New

Dept. of English Language \& Literature National University of Singapore

7 Arts Link, Block AS5

Singapore 117570

keelynzq@u.nus.edu
Michael Yoshitaka Erlewine

Dept. of English Language \& Literature

National University of Singapore

7 Arts Link, Block AS5

Singapore 117570

mitcho@nus.edu.sg 